\title{
A CASE REPORT
}

\author{
David Barbosa1, Nádia Simas², Sara Freire2 ${ }^{2}$ João Barreira², Emília Velhinho², Glória Nunes da Silva² \\ 1 - Endocrinology, Diabetes and Metabolism Department, Santa Maria Hospital, Lisbon, Portugal \\ 2 - Internal Medicine Department (3B Sector), Pulido Valente Hospital, Lisbon, Portugal
}

\section{Background}

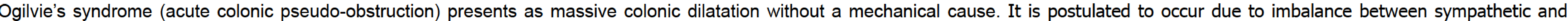
parasympathetic innervations of the large bowel, although the exact pathophysiology of intestinal pseudo-obstruction remains to be elucidated.

- Causes include:

$\checkmark$ Trauma, especially fractures; Obstetrical surgery, especially involving spinal anesthesia

$\checkmark$ Severe medical illness, such as pneumonia, myocardial infarction, or heart failure

$\checkmark$ Neurologic conditions

$\checkmark$ Metabolic imbalance (eg, Hypothyroidism, Hypokalaemia)

$\checkmark$ Medication administration (eg, narcotics, phenothiazines, calcium channel blockers, alpha-2-agonists)

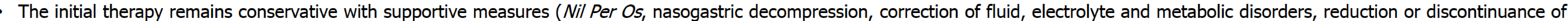

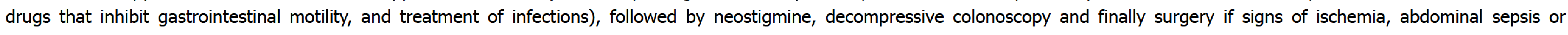
perforation are present.

- About $75 \%$ of cases resolve with conservative therapy in a median of 4 days

\section{Case Report}

Female, 51 years old, caucasian

PAST MEDICAL HISTORY

1. Hypothyroidism with poor adherence to therapy

2. Left subacromial bursitis

3. Status post plastic surgeries (lips and breasts)

4. Depressive syndrome

\section{PRESENT ILLNESS}

Abdominal colic pain, distension and obstipation, associated with nausea, puffiness of the eyes and adynamia for 1 month, aggravated in the last few days

\section{EXAMINATION}

At observation the patient was lethargic (GCS 14)

Abdomen was distended, with normal bowel sounds, diffusely painful to palpation but without signs of peritoneal involvement

BP: 128 / 74 mmHg; Pulse: 57 / min; Tympanic Temperature: $36.4{ }^{\circ} \mathrm{C}$

\begin{tabular}{|c|c|}
\hline \multicolumn{2}{|c|}{ LABORATORY EVALUATION } \\
\hline Variable & Value \\
\hline \multicolumn{2}{|l|}{ BIOCHEMISTRY } \\
\hline Urea $(\mathrm{mg} / \mathrm{dL})$ & 19 \\
\hline Creatinine (mg/dL) & 1.2 \\
\hline Creatine kinase $(\mathrm{U} / \mathrm{L})$ & 1252 \\
\hline $\mathrm{Na}^{+}(\mathrm{mmol} / \mathrm{L})$ & 141 \\
\hline $\mathrm{K}^{+}(\mathrm{mmol} / \mathrm{L})$ & 3.3 \\
\hline Glucose $(\mathrm{mg} / \mathrm{dL})$ & 88 \\
\hline PCR (mg/dL) & 0.9 \\
\hline \multicolumn{2}{|l|}{ ENDOCRINOLOGY } \\
\hline $\mathrm{TSH}(\mu \mathrm{g} / \mathrm{mL})$ & 80.8 \\
\hline $\mathrm{FT}_{4}(\mathrm{ng} / \mathrm{dL})$ & $<0.10$ \\
\hline Anti-TPO $(\mathrm{U} / \mathrm{mL})$ & $>1000$ \\
\hline Anti-TG (U/mL) & 36 \\
\hline Cortisol $(\mu \mathrm{g} / \mathrm{mL})$ & 14.9 \\
\hline
\end{tabular}

\section{Day 1}

HOSPITAL ADMISSION

Supportive measures + Hormone replacement with Levothyroxin

- Abdominal CT scan: distended bowel loops with a cutoff at the sigmoid colon, without any structural lesion visualized
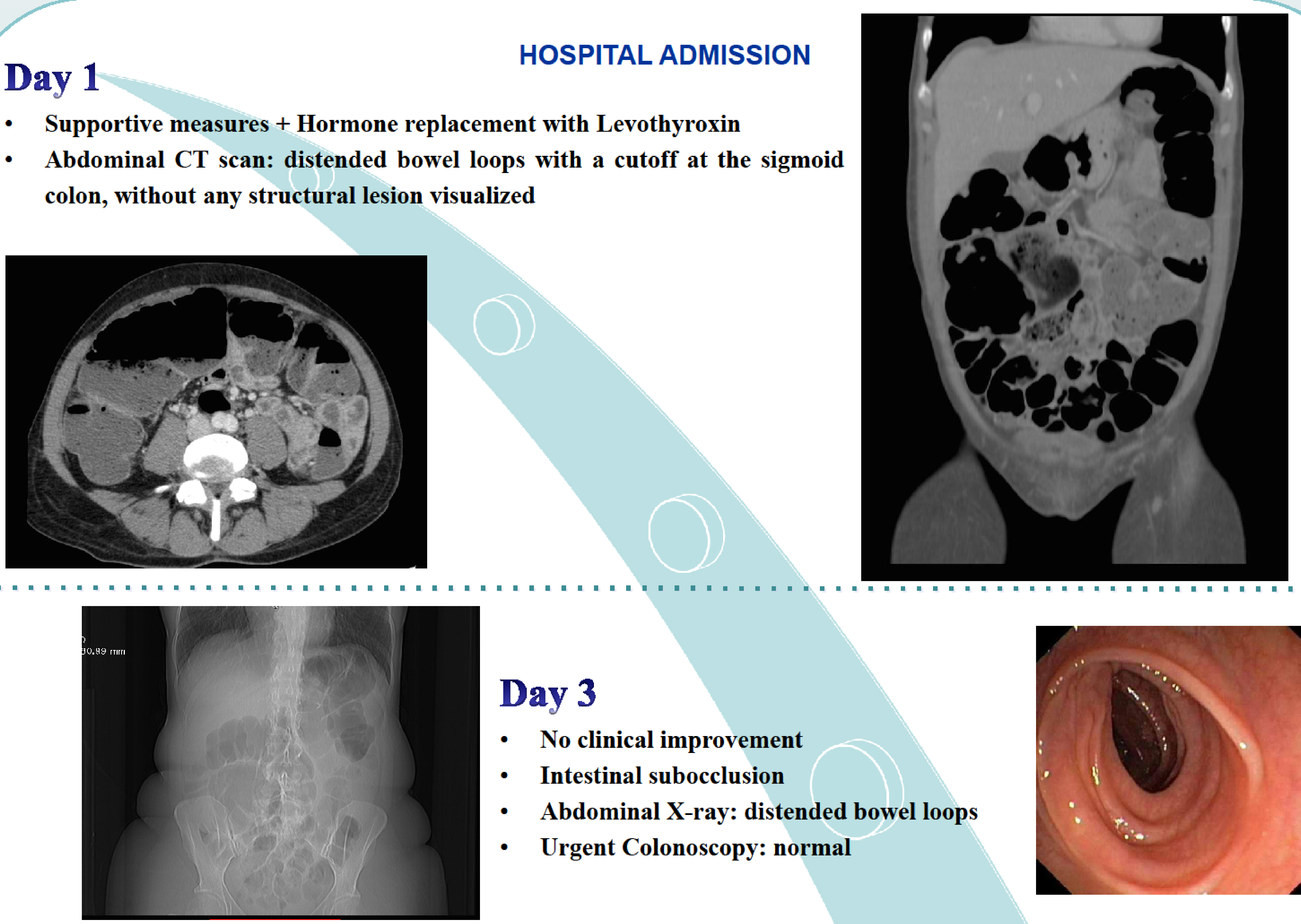

Day 3

- No clinical improvement

- Intestinal subocclusion

- Abdominal X-ray: distended bowel loops

Urgent Colonoscopy: normal

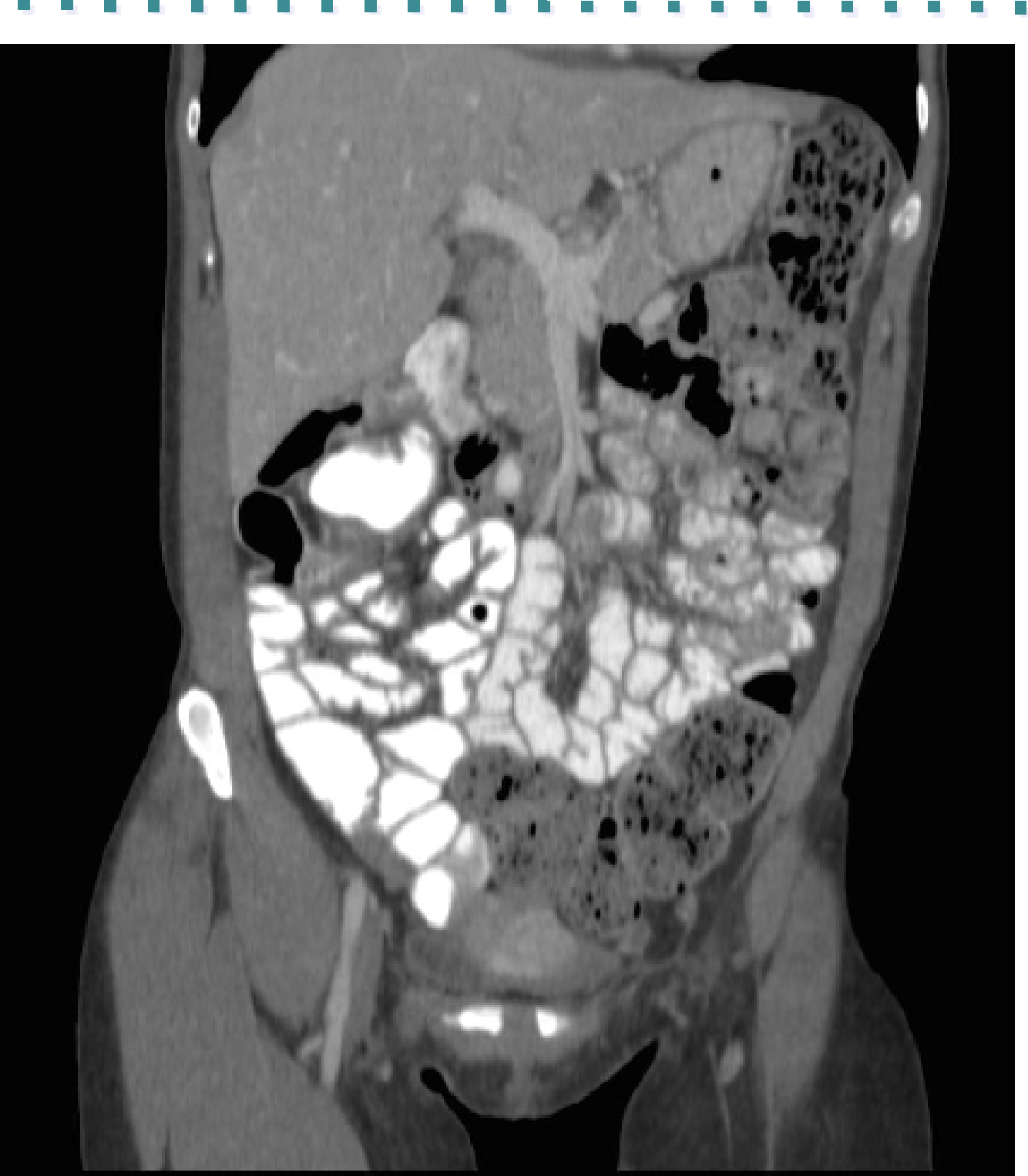

Day 5

- Clinical improvement, with subocclusion resolutio

- $2^{\text {nd }}$ Abdominal CT scan: normal

- Discharged

\section{Conclusion}

- We have described a patient with myxoedema crisis presenting as Ogilvie's syndrome, which is diagnosed only after excluding mechanical large bowel obstruction.

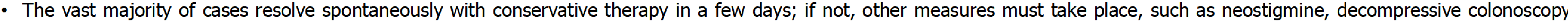
and finally surgery.

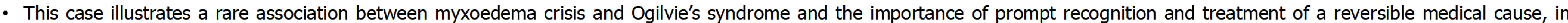
order to avoid other invasive measures. 